\title{
Gisting Continuous Speech
}

\author{
J. R. Rohlicek, Principal Investigator \\ BBN Systems and Technologies \\ 70 Fawcett St. \\ Cambridge, MA 02138
}

\section{PROJECT GOALS}

The objective of this work is automatic, real-time "gisting" of voice traffic for updating of information in databases, for producing timely reports, and for prompt notification of events of interest. Specifically, the goal is to build a prototype, real-time system capable of processing radio communication between air traffic controllers and pilots; identifying dialogs and extracting their "gist" (e.g., identifying flights, determining whether they are landing or taking off), and producing a continuous output stream with that information. The approach is intended to be general and applicable to other domains.

The system is built upon state-of-the-art techniques in speech recognition, speaker identification, natural language analysis, and topic statistical classification. These techniques have been extended where necessary to address specific aspects of the gisting problem. Because various sources of information must be combined, the system design features a high degree of interaction between the natural language and domain-knowledge components and the speech processing components.

\section{RECENT RESULTS}

We have made additions and modifications to our prototype system [1]. The primary goal of the effort was to achieve real-time performance. This involved both system architectural and algorithmic modifications described fully in [2].

A prototype system has been evaluated using approximately 14 hours of data recorded at Logan airport. Performance was measured on approximately four hours of data held out for final evaluation. On that data, the system achieved approximately $88 \%$ recall and $82 \%$ precision for detection of controller-pilot dialogs. Also, of the filights correctly detected, the fight identification was correctly extracted $59 \%$ of the time.

A real-time prototype system has been constructed. The system builds on a flexible software system developed as part of this effort. The system allows multiple processes to be coordinated across multiple hosts and provides facilities for efficient stream connections between modules as well as flexible message-based communication between modules.

\section{PLANS FOR THE COMING YEAR}

The remainder of the effort will focus on completion of the prototype system and on system testing. System testing includes investigation of the sensitivity of overall system performance to the performance of various component.

\section{REFERENCES}

[1] J. R. Rohlicek, et al, "Gisting conversational speech," Proceedings of the International Conference on Acoustics, Speech and Signal Processing, Mar 1992, pp. II-113.

[2] L. Denenberg, et. al., "Gisting conversational speech in real time," to appear in Proceedings of the International Conference on Acoustics, Speech and Signal Processing, April 1993. 